\title{
NÍVEIS DE MERCÚRIO TOTAL EM PEIXES DE ÁGUA DOCE \\ DE PISCICULTURAS PAULISTAS
}

\author{
Marcelo A. MORGANO ; Priscila C. GOMES , Dilza M. B. MANTOVANI ; \\ Adriana A. M. PERRONE ; Talita F. SANTOS
}

\begin{abstract}
RESUMO
Avaliou-se os niveis de mercúrio total em amostras de peixes de água doce, procedentes de pesqueiros e pisciculturas de 39 regiões do estado de São Paulo. As espécies de peixes estudadas foram: pacu (Piaractus mesopotamicus), tilápia (Oreochromis niloticus), piauçu (Leoporinum sp), matrinxã (Brycon cephalus), tambaqui (Colossoma macropomum) e carpa (Cyprinus carpis). Foram avaliadas três metodologias de digestão de amostras para a determinação de mercúrio total, variando-se o volume de mistura sulfonítrica utilizada e a concentração do permanganato de potássio, sendo que o uso de $10 \mathrm{~mL}$ de mistura sulfonítrica e solução de permanganato de potássio a $7 \%(\mathrm{~m} / \mathrm{v})$ na digestão da amostra foi a que forneceu as recuperações mais altas $(96 \%)$, precisão de $1 \%$ e limite de quantificação de $0,3 \mu \mathrm{g} / \mathrm{kg}$. Após a validação do método, foram realizadas as determinações de teor de mercúrio total em 293 amostras de pescado, usando a técnica de espectrometria de emissão com fonte de plasma acoplado em gerador de hidretos. Os niveis médios de mercúrio encontrados variaram entre: tambaqui $(0,0003-0,012 \mathrm{mg} / \mathrm{kg}), \mathrm{carpa}(0,063 \mathrm{mg} / \mathrm{kg}), \mathrm{matrinxã}$ $(0,0003-0,074 \mathrm{mg} / \mathrm{kg})$, pacu $(0,0003-0,078 \mathrm{mg} / \mathrm{kg})$, piauçu $(0,0003-0,183 \mathrm{mg} / \mathrm{kg})$ e tilápia $(0,0003-0,217 \mathrm{mg} / \mathrm{kg})$. Os resultados mostraram que nenhuma das amostras procedentes das diferentes regiões e diferentes pesque-pagues, apresentaram niveis de mercúrio total acima do permitido pela legislação brasileira, que é de $0,5 \mathrm{mg} / \mathrm{kg}$ para pescado não-predador.
\end{abstract}

Palavras-chave: peixe; mercúrio; determinação; espectrometria de emissão; gerador de hidreto.

\section{SUMMARY}

MERCURY LEVELS IN FRESHWATER FISHES FROM PISCICULTURES ESTABILISHED IN SÃO PAULO STATE. In this work, the levels of mercury were evaluated in samples of freshwater fish, coming from fishing-grounds and piscicultures of 39 regions of São Paulo State. The species of fish studied were: pacu (Piaractus mesopotamicus), tilápia (Oreochromis niloticus), piauçu (Leoporinum sp), matrinxã (Brycon cephalus), tambaqui (Colossoma macropomum) and carpa (Cyprinus carpis). Three digestion methodologies of sample were evaluated for the mercury determination, with variation of the volume of sulphonitric mixture utilized and the potassium permanganate concentration. After the validation method the determinations of mercury level were recorded in 293 samples of fish, using the technique of emission spectrometry with plasma coupled to an hydrides generator. The medium levels of mercury found were the following: tambaqui $(0,0003-0,012 \mathrm{mg} / \mathrm{kg})$, carpa $(0,063 \mathrm{mg} / \mathrm{kg})$, matrinxã $(0,0003-0,074 \mathrm{mg} / \mathrm{kg}), \mathrm{pacu}$ $(0,0003-0,078 \mathrm{mg} / \mathrm{kg})$, piauçu $(0,0003-0,183 \mathrm{mg} / \mathrm{kg})$ and tilápia $(0,0003-0,217 \mathrm{mg} / \mathrm{kg})$, and this last one presented the higher contamination rate. The results showed that none of the samples obtained from the different regions and fish-and-pay establishments presented mercury levels above the permitted limits of Brazilian legislation; 0,5mg/ $\mathrm{kg}$ for non carnivorous fish.

Keywords: fish; mercury; determination; emission spectrometry; hydride generator.

\section{1 - INTRODUÇÃO}

A produção de pescado vem crescendo nos últimos anos, juntamente com o desenvolvimento da criação de organismos aquáticos. No estado de São Paulo, há um grande número de produtores motivados a investir nesse setor, devido à grande disponibilidade de recursos produtivos e ao grande mercado promissor. O crescimento rápido pode levar ao descontrole, o que gera preocupação do ponto de vista técnico. A falta de uma orientação médico-veterinária adequada leva ao uso incorreto de produtos químicos na cura de enfermidades de peixes. A criação de peixes em áreas próximas às de cultivo agrícola, onde há uso exagerado e incorreto de agrotóxicos, principalmente os organomercuriais, poderá ser contaminada [12]. Substâncias químicas presentes nesses produtos

Recebido para publicação em 25/04/2003. Aceito para publicação em 19/04/2005(001119).

Centro de Química e Nutrição Aplicada, Instituto de Tecnologia de Alimentos (ITAL). End.: Av. Brasil, 2880. CEP: 13073-001, Campinas, SP. End. Eletr.: http://www.ital.org.br

A quem a correspondência deve ser enviada. são geralmente metais pesados, como por exemplo o mercúrio, e podem bioacumular-se ao longo da cadeia alimentar. Os metais pesados, quando em excesso, são tóxicos e podem causar danos ao organismo humano, principalmente alterações no sistema nervoso central. Contudo, há necessidade de maiores investigações por parte dos órgãos de pesquisa e, também, avaliação da real necessidade do uso desses produtos.

O peixe tem sido apontado como a principal via de intoxicação do ser humano por mercúrio. A forma química mais tóxica do mercúrio tem sido identificada como a do metilmercúrio [9]. O mercúrio é transformado em metilmercúrio por ação de bactérias; o peixe absorve o metilmercúrio da água e, também, pela ingestão de organismos aquáticos [13]. Mesmo em regiões com niveis normais de mercúrio na água, podem ser observados níveis altos de mercúrio em peixes, pois ao ser incorporado na cadeia trófica, o mercúrio é biomagnificado e bioacumulado, devido a sua longa meia-vida nos organismos (640 a 1200 dias) [11]. No homem, a absorção intestinal do metilmercúrio é maior que $95 \%$ e sua meia-vida biológica para eliminação é em torno de 70 dias. Quando é absorvido, acumula-se nos rins, no figado e no sistema nervoso cen- 
tral (SNC), atuando como inibidor enzimático, inativando proteinas pelo bloqueio de radicais $\mathrm{SH}$ [14].

As manifestações clínicas da intoxicação por mercúrio podem ser agudas ou crônicas. A aguda, devido à ingestão de alimentos contaminados por metilmercúrio, produz um quadro que varia de leve a letal, com vômitos freqüentes, tremores, ataxia, parestesia, paralisia, afonia, cegueira, coma e morte. A intoxicação crônica afeta principalmente o SNC, causando parestesia, ataxia, dificuldade de articular palavras, sensação generalizada de fraqueza, fadiga e incapacidade de concentração, perda de visão e audição, coma e morte [14]. Os efeitos clínicos não são imediatos. No caso de exposição ambiental, o período latente pode chegar a 10 anos e será inversamente proporcional ao nível de exposição. O metilmercúrio tem efeito feto-tóxico, com alterações irreversiveis para o feto, mesmo sem o aparecimento de sintomas na mãe. Os sintomas observados em neonatais e crianças, devido à exposição pré-natal, são a paralisia cerebral, distúrbios mentais, retardamento do desenvolvimento de várias funções psicomotoras, convulsões, cegueira e má-formação dos ouvidos [1].

O risco de intoxicação pela ingestão de peixe contaminado depende de alguns fatores, sendo que o teor de mercúrio, a quantidade ingerida e a freqüência de ingestão são determinantes para a acumulação de metilmercúrio no organismo humano [1]. Visando assegurar a Saúde Pública, foram estabelecidos limites de tolerância de mercúrio em alimentos. Para pescados, o Brasil fixou a tolerância em $0,5 \mathrm{mg} / \mathrm{kg}$, para pescado não-predador, e $1,0 \mathrm{mg} / \mathrm{kg}$ para pescado predador [2].

O uso de espectrometria de emissão com fonte de plasma (ICP OES) acoplado a gerador de hidretos na análise de mercúrio, tem sido bastante difundido, por se tratar de uma técnica inovadora e que apresenta alta sensibilidade para o metal e boa reprodutibilidade dos resultados [7]. A técnica é muito bem sucedida em função da capacidade de análises multielementares e de elementos traços em diversos tipos de amostra [8].

O objetivo do trabalho foi validar uma metodologia de digestão de amostra para a determinação de mercúrio total em peixes usando a técnica de espectrometria de emissão com fonte de plasma, acoplado em gerador de hidretos, e verificar a possível contaminação por mercúrio total em diferentes espécies e amostras de peixes, procedentes de pesque-pagues e pisciculturas do estado de São Paulo.

\section{2 - MATERIAL E MÉTODOS}

\section{1 - Material}

Para a quantificação do mercúrio em peixes, foram coletadas amostras de pacu (Piaractus mesopotamicus), tilápia (Oreochromis niloticus), piauçu (Leoporinum sp), matrinxã (Brycon cephalus), tambaqui (Colossoma macropomum) e carpa (Cyprinus carpis) em pesqueiros de diferentes regiões do estado de São Paulo, totalizando 39 re- giões, no período de setembro/2001 a maio/2002, num total de 293 amostras. Para cada pesqueiro foram analisadas de duas a quatro espécies diferentes de peixe ( $\mathrm{Ta}$ bela 3) dependendo da disponibilidade das espécies na época de coleta das amostras.

De cada amostra separou-se o filé das espinhas, escamas e vísceras. Posteriormente, o filé foi homogeneizado e armazenado a $18^{\circ} \mathrm{C}$ até o momento da análise.

\section{2 - Métodos}

\subsection{1 - Digestão da amostra}

O método de digestão usado foi adaptado do método proposto por YALLOUZ \& CAMPOS [15]. Nos testes realizados, variou-se o volume de mistura sulfonítrica $\left(\mathrm{HNO}_{2}: \mathrm{H}_{2} \mathrm{SO}_{1}, 1: 1\right)$ com $0,1 \%$ de pentóxido de vanádio $\left(\mathrm{V}_{2} \mathrm{O}_{s}\right)$ e a concentração de permanganato de potássio $(\mathrm{KMNO}$, ). Para a digestão, foram pesados $0,5 \mathrm{~g}$ de amostra diretamente no tubo de digestão e, em seguida, foram adicionados $10 \mathrm{~mL}$ de mistura sulfonitrica $\left(\mathrm{HNO}_{2}: \mathrm{H}_{2} \mathrm{SO}\right.$ $1: 1)$ com $0,1 \%(\mathrm{~m} / \mathrm{v}) \mathrm{V}$ O e deixado em repouso por aproximadamente 12 horas. Após o repouso, a mistura foi submetida a aquecimento em banho-maria a $90^{\circ} \mathrm{C} \mathrm{du}-$ rante 3 horas. Em seguida foi adicionado $5 \mathrm{~mL}$ de $\mathrm{KMNO}$. $7 \%(\mathrm{~m} / \mathrm{v})$ e submeteu-se a mistura a aquecimento em banho-maria a $90^{\circ} \mathrm{C}$ durante 3 horas. O excesso de oxidante foi reduzido com $900 \mu \mathrm{L}$ de solução de cloridrato de hidroxilamina $20 \%(\mathrm{~m} / \mathrm{v})$. O conteúdo do tubo foi transferido para balão volumétrico de $50 \mathrm{~mL}$, completando o volume com água bidestilada.

\subsection{2 - Determinação do mercúrio}

Para determinar o mercúrio total presente nas amostras estudadas, utilizou-se a técnica da Espectrometria de Emissão Óptica com Fonte de Plasma Indutivamente Acoplado (ICP OES), com uso do espectrômetro simultâneo VARIAN modelo VISTA-MPX (Mulgrave, Austrália) e gerador de hidretos VARIAN-VGA77 (Mulgrave, Austrália). As condições de operação do aparelho foram: potência-1000Watts, fluxo do gás Argônio- $15 \mathrm{~L} / \mathrm{min}$, fluxo do gás auxiliar-1,5L/min, tempo de replicata-10s, tempo de estabilização-15s, tempo de limpeza-10s e comprimento de onda para leitura do mercúrio-253,652nm.

\subsection{3 - Validação do método}

Uma amostra de filé de peixe homogeneizado foi usada como matriz para a validação do método $[4,5]$ de determinação de mercúrio total. A exatidão foi verificada pela avaliação da recuperação de mercúrio adicionado à amostra (Tabela 2). A curva de calibração na faixa de 0,5 a $50 \mu \mathrm{g} / \mathrm{L}$ foi linear em toda faixa de concentração $(\mathrm{r}=$ 0,9999).

\section{3 - RESULTADOS E DISCUSSÃO}

Nos testes realizados para a validação do método, onde foram variados o volume de mistura sulfonitrica e a concentração da solução de permanganato de potássio, o 
que apresentou recuperação mais alta (96\%) foi o teste 03 , onde se usou $10 \mathrm{~mL}$ de mistura sulfonitrica e concentração de permanganato de potássio igual a 7\% (Tabela 1). Estes testes indicaram que é critica a escolha das condições ácido/oxidante durante a digestão da amostra e que amostras parcialmente digeridas podem fornecer resultados inferiores ao valor real da amostra.

TABELA 1 - Resultados obtidos nos testes para a escolha das condições do sistema ácido/oxidante para digestão da amostra

\begin{tabular}{cccc}
\hline Teste & $\begin{array}{c}\text { Volume de mistura } \\
\text { sulfonitrica }(\mathrm{mL})\end{array}$ & $\begin{array}{c}\text { Concentração de } \\
\mathrm{KMNO}_{4} \%(\mathrm{~m} / \mathrm{v})\end{array}$ & \% de recuperação \\
\hline 01 & 10 & 5 & 78 \\
02 & 15 & 10 & 43 \\
03 & 10 & 7 & 96 \\
\hline
\end{tabular}

A precisão do método definida pelo coeficiente de variação observado foi de 1,0\%. O limite de detecção encontrado foi de $0,1 \mu \mathrm{g} / \mathrm{kg}$ (3 vezes o desvio padrão de 10 repetições de branco) e o limite de quantificação de $0,3 \mu \mathrm{g} / \mathrm{kg}$ (10 vezes o desvio padrão de 10 repetições de branco). Os parâmetros ensaiados indicaram adequação do método empregado.

TABELA 2 - Resultados obtidos na avaliação da metodologia, usando teste de recuperação em amostra de peixe, com adição de solução padrão do mercúrio em três niveis diferentes de concentração

\begin{tabular}{cccc}
\hline $\begin{array}{c}\text { Quantidade } \\
\text { adicionada } \\
(\mathrm{mg} / \mathrm{kg})\end{array}$ & $\begin{array}{c}\text { Resultado obtido* } \\
(\mathrm{mg} / \mathrm{kg})\end{array}$ & $\begin{array}{c}\text { Valor esperado } \\
(\mathrm{mg} / \mathrm{kg})\end{array}$ & $\begin{array}{c}\text { Porcentagem de } \\
\text { recuperação* }(\%)\end{array}$ \\
\hline 0,020 & $0,019 \pm 0,001$ & 0,020 & $94 \pm 3$ \\
0,030 & $0,029 \pm 0,001$ & 0,030 & $96 \pm 2$ \\
0,040 & $0,039 \pm 0,001$ & 0,040 & $98 \pm 1$ \\
\hline
\end{tabular}

* mèdia de trēs repetiçōes analiticas + estimativa de desvio padrāo.

As espécies analisadas eram de pescado nãopredador, e apresentaram concentrações de mercúrio total (Tabela 3) abaixo dos limites estabelecidos pela legislação brasileira vigente (Portaria no 685/98), de $0,5 \mathrm{mg} / \mathrm{kg}$ para pescado não-predador [2], correspondendo à metade do valor permitido. Resultados encontrados acima do limite permitido pela legislação brasileira vigente, poderiam indicar contaminação dos pesqueiros do estado de São Paulo. Não foi confirmada a expectativa de contaminação por mercúrio total nas espécies analisadas.

Verificou-se que o tambaqui apresentou menor nível de contaminação por mercúrio $(0,0003-0,012 \mathrm{mg} / \mathrm{kg})$ do que a carpa $(0,063 \mathrm{mg} / \mathrm{kg})$, matrinxã $(0,0003$ $0,074 \mathrm{mg} / \mathrm{kg})$, pacu $(0,0003$ - 0,078mg $/ \mathrm{kg})$, piauçu $(0,0003-0,183 \mathrm{mg} / \mathrm{kg})$. A tilápia $(0,0003-0,217 \mathrm{mg} / \mathrm{kg})$ apresentou o maior índice de contaminação.

Nas amostras das regiões de Barretos, Catanduva e Registro, o nivel de mercúrio total encontra-se abaixo do limite de quantificação, o que não foi observado nas outras regiões, onde as amostras apresentaram niveis acima do limite de quantificação. Na região de Sorocaba, foi encontrada a maior contaminação em relação às outras regiões analisadas, $0,217 \mathrm{mg} / \mathrm{kg}$ em uma espécie de tilápia.
KITAHARA et al. [9] analisaram amostras de peixes de diferentes locais de captura e de diferentes procedências comerciais do país inteiro. Dentre as espécies analisadas, apenas a tilápia e o matrinxã coincidiram com as espécies analisadas no presente trabalho. Esse autores encontraram 0,01 - 0,02mg/kg para tilápia e 0,04 $0,26 \mathrm{mg} / \mathrm{kg}$ para matrinxã. FUMOTO \& OLIVEIRA [6] analisaram peixes de diferentes espécies marinhos e de água doce comercializados em São Paulo - SP e encontrando níveis de mercúrio variando de $0,02-0,06 \mathrm{mg} / \mathrm{kg}$ para tilápia. DAMATO, SÃO CLEMENTE \& SANTOS [3] analisaram mexilhões procedentes da Baixada Fluminense e encontraram níveis médios de mercúrio que variaram de 0,03 a 0,06mg/kg. No trabalho de PEREIRA et al. [10], foi

TABELA 3 - Teor de mercúrio encontrado nas amostras de peixes coletadas em diferentes regiões do estado de São Paulo e em diferentes pesque-pagues

\begin{tabular}{|c|c|c|c|c|c|}
\hline \multirow[t]{2}{*}{ Região } & \multirow[t]{2}{*}{ Espécie } & \multicolumn{4}{|c|}{$\mathrm{Hg}(\mathrm{mg} / \mathrm{kg})$} \\
\hline & & $\begin{array}{l}\text { Pesque } \\
\text { pague I }\end{array}$ & $\begin{array}{l}\text { Pesque } \\
\text { pague II }\end{array}$ & $\begin{array}{c}\text { Pesque } \\
\text { pague III }\end{array}$ & $\begin{array}{c}\text { Pesque } \\
\text { pague IV }\end{array}$ \\
\hline Andradina & $\begin{array}{l}\text { pacu } \\
\text { tilápia } \\
\text { matrinxã }\end{array}$ & $\begin{array}{c}0,005 \\
<0,0003 \\
0,024\end{array}$ & $\begin{array}{l}<0,0003 \\
<0,0003\end{array}$ & $\begin{array}{l}<0,0003 \\
<0,0003\end{array}$ & \\
\hline Araçatuba & $\begin{array}{l}\text { pacu } \\
\text { tilápia }\end{array}$ & $\begin{array}{l}0,028 \\
0,034\end{array}$ & $\begin{array}{l}0,033 \\
0,046\end{array}$ & $\begin{array}{l}0,010 \\
0,037\end{array}$ & $\begin{array}{l}0,028 \\
0,032\end{array}$ \\
\hline Araraquara & $\begin{array}{l}\text { pacu } \\
\text { tilápia }\end{array}$ & $\begin{array}{l}<0,0003 \\
<0,0003\end{array}$ & $\begin{array}{c}<0,0003 \\
0,004\end{array}$ & $<0,0003$ & $<0,0003$ \\
\hline Assis & $\begin{array}{l}\text { pacu } \\
\text { tilápia } \\
\text { piauçu } \\
\text { matrinxã }\end{array}$ & $\begin{array}{l}0,032 \\
0,065 \\
0,041 \\
0,074\end{array}$ & $\begin{array}{l}0,038 \\
0,037 \\
0,053\end{array}$ & $\begin{array}{l}0,065 \\
0,062\end{array}$ & 0,065 \\
\hline Avaré & $\begin{array}{l}\text { pacu } \\
\text { tilápia } \\
\text { piauçu }\end{array}$ & $\begin{array}{l}0,023 \\
0,038 \\
0,008 \\
\end{array}$ & $\begin{array}{l}0,012 \\
0,013\end{array}$ & $\begin{array}{l}0,015 \\
0,017\end{array}$ & 0,013 \\
\hline Barretos & $\begin{array}{l}\text { pacu } \\
\text { tilápia } \\
\text { matrinxã }\end{array}$ & $\begin{array}{l}<0,0003 \\
<0,0003 \\
<0,0003\end{array}$ & $\begin{array}{l}<0,0003 \\
<0,0003\end{array}$ & $\begin{array}{l}<0,0003 \\
<0,0003\end{array}$ & $<0,0003$ \\
\hline Bauru & $\begin{array}{l}\text { pacu } \\
\text { tilápia } \\
\text { piaucu }\end{array}$ & $\begin{array}{l}0,003 \\
0,013 \\
0,183 \\
\end{array}$ & $\begin{array}{l}0,078 \\
0,005 \\
0,147 \\
\end{array}$ & $\begin{array}{l}<0,0003 \\
<0,0003 \\
<0,0003\end{array}$ & $\begin{array}{l}0,002 \\
0,004\end{array}$ \\
\hline Botucatu & $\begin{array}{l}\text { pacu } \\
\text { tilápia } \\
\text { piauçu }\end{array}$ & $\begin{array}{l}0,026 \\
0,024 \\
0,039 \\
\end{array}$ & $\begin{array}{l}0,024 \\
0,038\end{array}$ & $\begin{array}{l}0,040 \\
0,037\end{array}$ & $\begin{array}{l}0,028 \\
0,036\end{array}$ \\
\hline Bragança Paulista & $\begin{array}{l}\text { pacu } \\
\text { tilápia }\end{array}$ & $\begin{array}{l}0,039 \\
0,039\end{array}$ & $\begin{array}{l}0,029 \\
0,183\end{array}$ & $\begin{array}{l}0,027 \\
0,043\end{array}$ & $\begin{array}{l}0,040 \\
0,046\end{array}$ \\
\hline Campinas & $\begin{array}{l}\text { pacu } \\
\text { tilápia } \\
\text { piauçu } \\
\text { matrinxã }\end{array}$ & $\begin{array}{l}0,016 \\
0,015 \\
0,014 \\
0,006\end{array}$ & $\begin{array}{l}0,012 \\
0,007\end{array}$ & $\begin{array}{c}<0,0003 \\
0,005\end{array}$ & $\begin{array}{c}<0,0003 \\
0,023\end{array}$ \\
\hline Catanduva & $\begin{array}{l}\text { pacu } \\
\text { tilápia } \\
\text { matrinxã }\end{array}$ & $\begin{array}{l}<0,0003 \\
<0,0003 \\
<0,0003\end{array}$ & $\begin{array}{l}<0,0003 \\
<0,0003 \\
<0,0003\end{array}$ & $<0,0003$ & $<0,0003$ \\
\hline Dracena & $\begin{array}{l}\text { pacu } \\
\text { tilápia } \\
\text { piauçu } \\
\text { matrinxã }\end{array}$ & $\begin{array}{l}0,010 \\
0,003 \\
0,006 \\
0,016\end{array}$ & $\begin{array}{l}<0,0003 \\
<0,0003\end{array}$ & 0,002 & $<0,0003$ \\
\hline Fernandópolis & $\begin{array}{l}\text { pacu } \\
\text { tilápia } \\
\text { piauçu } \\
\text { matrinxã }\end{array}$ & $\begin{array}{l}0,033 \\
0,028 \\
0,041 \\
0,044\end{array}$ & $\begin{array}{l}0,021 \\
0,016\end{array}$ & $\begin{array}{l}0,020 \\
0,025\end{array}$ & \\
\hline Franca & $\begin{array}{l}\text { pacu } \\
\text { tilápia }\end{array}$ & $\begin{array}{c}<0,0003 \\
0,018\end{array}$ & $\begin{array}{c}<0,0003 \\
0,017\end{array}$ & 0,005 & 0,005 \\
\hline General Salgado & $\begin{array}{l}\text { pacu } \\
\text { tilápia } \\
\text { piauçu }\end{array}$ & $\begin{array}{c}<0,0003 \\
0,007 \\
0,002 \\
\end{array}$ & $<0,0003$ & $<0,0003$ & $<0,0003$ \\
\hline Guaratinguetá & $\begin{array}{l}\text { pacu } \\
\text { tilápia } \\
\text { piauçu }\end{array}$ & $\begin{array}{c}<0,0003 \\
0,010 \\
0,026\end{array}$ & $\begin{array}{c}<0,0003 \\
0,004\end{array}$ & 0,004 & $<0,0003$ \\
\hline Itapeva & $\begin{array}{l}\text { pacu } \\
\text { tilápia }\end{array}$ & $\begin{array}{l}0,006 \\
0,009\end{array}$ & $\begin{array}{c}<0,0003 \\
0,004\end{array}$ & 0,006 & 0,002 \\
\hline Jaboticabal & $\begin{array}{l}\text { pacu } \\
\text { tilápia } \\
\text { piauçu } \\
\text { matrinxã }\end{array}$ & $\begin{array}{c}<0,0003 \\
0,029 \\
<0,0003 \\
0,011\end{array}$ & $\begin{array}{c}<0,0003 \\
0,017 \\
<0,0003\end{array}$ & $<0,0003$ & \\
\hline Jales & $\begin{array}{l}\text { pacu } \\
\text { piauçu }\end{array}$ & $\begin{array}{l}<0,003 \\
<0,003\end{array}$ & $<0,003$ & $<0,003$ & $<0,003$ \\
\hline Jaú & $\begin{array}{l}\text { pacu } \\
\text { tilápia } \\
\text { piaucucu }\end{array}$ & $\begin{array}{c}0,002 \\
0,015 \\
<0,0003\end{array}$ & $\begin{array}{c}0,009 \\
0,004 \\
<0,0003 \\
\end{array}$ & $\begin{array}{l}0,003 \\
0,011\end{array}$ & $<0,0003$ \\
\hline Limeira & $\begin{array}{l}\text { pacu } \\
\text { tilápia } \\
\text { matrinxã }\end{array}$ & $\begin{array}{l}0,023 \\
0,018 \\
0,014\end{array}$ & $\begin{array}{l}0,007 \\
0,018 \\
0,021\end{array}$ & $\begin{array}{l}0,031 \\
0,018\end{array}$ & \\
\hline
\end{tabular}




\begin{tabular}{|c|c|c|c|c|c|}
\hline Lins & $\begin{array}{l}\text { pacu } \\
\text { tilápia } \\
\text { piauçu }\end{array}$ & $\begin{array}{c}<0,0003 \\
0,008 \\
0,010\end{array}$ & $\begin{array}{c}<0,0003 \\
<0,0003 \\
0,012\end{array}$ & & \\
\hline Marília & $\begin{array}{l}\text { pacu } \\
\text { tilápia }\end{array}$ & $\begin{array}{l}0,018 \\
0,017\end{array}$ & $\begin{array}{l}0,022 \\
0,019\end{array}$ & $\begin{array}{l}0,024 \\
0,020\end{array}$ & 0,015 \\
\hline Mogi das Cruzes & $\begin{array}{l}\text { pacu } \\
\text { tilápia } \\
\text { matrinxã }\end{array}$ & $\begin{array}{l}0,041 \\
0,025 \\
0,030\end{array}$ & $\begin{array}{l}0,030 \\
0,022\end{array}$ & $\begin{array}{l}0,024 \\
0,029\end{array}$ & 0,043 \\
\hline Mogi Mirim & $\begin{array}{l}\text { pacu } \\
\text { tilápia } \\
\text { matrinxã }\end{array}$ & $\begin{array}{l}0,050 \\
0,046 \\
0,028 \\
\end{array}$ & $\begin{array}{l}0,023 \\
0,033 \\
0,050 \\
\end{array}$ & & \\
\hline Orlândia & $\begin{array}{l}\text { pacu } \\
\text { tilápia } \\
\text { piauçu } \\
\text { matrinxã }\end{array}$ & $\begin{array}{c}<0,0003 \\
0,005 \\
<0,0003 \\
<0,0003\end{array}$ & $\begin{aligned}< & 0,0003 \\
& 0,005 \\
< & 0,0003 \\
< & 0,0003\end{aligned}$ & $\begin{array}{l}<0,0003 \\
<0,0003 \\
<0,0003\end{array}$ & $<0,0003$ \\
\hline Ourinhos & $\begin{array}{l}\text { pacu } \\
\text { tilápia } \\
\text { piauçu } \\
\text { matrinxã }\end{array}$ & $\begin{array}{c}<0,0003 \\
<0,0003 \\
<0,0003 \\
0,008\end{array}$ & $\begin{array}{c}<0,0003 \\
<0,0003 \\
0,035\end{array}$ & $\begin{array}{l}0,007 \\
0,066\end{array}$ & $<0,0003$ \\
\hline Pindamonhangaba & $\begin{array}{l}\text { pacu } \\
\text { tilápia }\end{array}$ & $\begin{array}{c}<0,0003 \\
0,089\end{array}$ & $\begin{array}{c}0,006 \\
<0,0003\end{array}$ & $\begin{array}{c}<0,0003 \\
0,003\end{array}$ & $\begin{array}{l}<0,0003 \\
<0,0003\end{array}$ \\
\hline Piracicaba & $\begin{array}{l}\text { pacu } \\
\text { tilápia } \\
\text { matrinxã }\end{array}$ & $\begin{array}{l}0,010 \\
0,016 \\
0,019 \\
\end{array}$ & $\begin{array}{l}0,003 \\
0,011\end{array}$ & $\begin{array}{l}0,010 \\
0,005\end{array}$ & 0,020 \\
\hline $\begin{array}{l}\text { Presidente } \\
\text { Prudente }\end{array}$ & $\begin{array}{l}\text { pacu } \\
\text { tilápia } \\
\text { piauçu }\end{array}$ & $\begin{array}{l}0,005 \\
0,005 \\
0,026\end{array}$ & $\begin{array}{l}0,011 \\
0,017\end{array}$ & $\begin{array}{c}0,012 \\
<0,0003\end{array}$ & $<0,0003$ \\
\hline $\begin{array}{l}\text { Presidente } \\
\text { Venceslau }\end{array}$ & $\begin{array}{l}\text { pacu } \\
\text { tilápia }\end{array}$ & $\begin{array}{c}<0,0003 \\
0,019\end{array}$ & $\begin{array}{l}0,011 \\
0,004\end{array}$ & $\begin{array}{c}0,012 \\
<0,0003\end{array}$ & $<0,0003$ \\
\hline Registro & $\begin{array}{l}\text { pacu } \\
\text { tilápia }\end{array}$ & $\begin{array}{l}<0,0003 \\
<0,0003\end{array}$ & & & \\
\hline Ribeirão Preto & $\begin{array}{l}\text { pacu } \\
\text { tilápia } \\
\text { piauçu } \\
\text { tambaqui }\end{array}$ & $\begin{array}{l}<0,0003 \\
<0,0003 \\
<0,0003 \\
<0,0003\end{array}$ & $\begin{array}{l}<0,0003 \\
<0,0003\end{array}$ & 0,002 & $<0,0003$ \\
\hline $\begin{array}{l}\text { São João da Boa } \\
\text { Vista }\end{array}$ & $\begin{array}{l}\text { pacu } \\
\text { tilápia } \\
\text { carpa }\end{array}$ & $\begin{array}{l}0,001 \\
0,005 \\
0,013\end{array}$ & $\begin{array}{c}<0,0003 \\
0,007\end{array}$ & $\begin{array}{c}<0,0003 \\
0,006\end{array}$ & \\
\hline $\begin{array}{l}\text { São José do Rio } \\
\text { Preto }\end{array}$ & $\begin{array}{l}\text { pacu } \\
\text { piauçu } \\
\text { tambaqui }\end{array}$ & $\begin{array}{c}<0,0003 \\
<0,0003 \\
0,006\end{array}$ & 0,006 & & \\
\hline São Paulo & $\begin{array}{l}\text { pacu } \\
\text { tilápia } \\
\text { piauçu } \\
\text { tambaqui }\end{array}$ & $\begin{array}{c}<0,0003 \\
0,024 \\
0,016 \\
0,012\end{array}$ & $\begin{array}{c}<0,0003 \\
0,005\end{array}$ & $<0,0003$ & $<0,0003$ \\
\hline Sorocaba & $\begin{array}{l}\text { pacu } \\
\text { tilápia }\end{array}$ & $\begin{array}{l}0,034 \\
0,019\end{array}$ & $\begin{array}{l}0,035 \\
0,217\end{array}$ & $\begin{array}{l}0,074 \\
0,063\end{array}$ & 0,031 \\
\hline Tupã & $\begin{array}{l}\text { pacu } \\
\text { tilápia }\end{array}$ & $\begin{array}{l}0,012 \\
0,013\end{array}$ & $\begin{array}{l}0,008 \\
0,004\end{array}$ & $\begin{array}{l}0,016 \\
0,013\end{array}$ & 0,013 \\
\hline Votuporanga & $\begin{array}{l}\text { pacu } \\
\text { tilápia } \\
\text { piaucucu }\end{array}$ & $\begin{array}{l}0,015 \\
0,010 \\
0,019\end{array}$ & $\begin{array}{l}0,019 \\
0,012\end{array}$ & $<0,0003$ & \\
\hline
\end{tabular}

encontrado níveis de mercúrio variando de 0,02 a $0,04 \mathrm{mg} / \mathrm{kg}$ em amostras de moluscos bivalves coletados no litoral da Baixada Santista. De um modo geral, esses resultados estão próximos aos apresentados na Tabela 3.

\section{4 - CONCLUSÕES}

A validação da metodologia, evidenciou que na etapa de digestão da amostra a quantidade de ácido e oxidante empregado é o ponto crítico e que o uso da técnica de ICP OES acoplada a gerador de hidretos pode fornecer resultados confiáveis e limites de quantificação adequados para a determinação de mercúrio em amostras de peixes.

Podemos concluir que, no período estudado, as amostras de peixes procedentes de pesqueiros e pisciculturas das diferentes regiões do estado de São Paulo, encontravam-se em condições adequadas para o consumo humano, pois todas as amostras analisadas apresentaram níveis de mercúrio total inferiores ao estabelecido pela legislação brasileira e do MERCOSUL em vigor.

Não foi encontrada nenhuma relação para a concentração de mercúrio total entre o local de coleta e a espécie analisada.

\section{5 - REFERÊNCIAS BIBLIOGRÁFICAS}

[1] ATSDR (Agency for Toxic Substances and Disease Registry), 1989. Toxicological profile for mercury. Atlanta, U. S. Public Health Service.

[2] BRASIL, LEIS, DECRETOS, etc. Divisão Nacional de Vigilância Sanitária de Alimentos DINAL - Portaria $n^{\circ}$ 685 de 27 de agosto de 1998. Diário Oficial da União. Brasília. seç.1, pt.1, p.1415-1437, 24 set. 1998.

[3] DAMATO, C.; SÃO CLEMENTE, S.C.; SANTOS, N.N. Levantamento sobre os índices de mercúrio presentes em mexilões (Perna perna L.) oriundos da Praia de Piratininga e Baía de Quanabara e Sepitiba. Higiene Alimentar, v. 11, n. 50, p. 30-35, 1997.

[4] FDA (Food and Drug Administration), 2000. Guidance for Industry. Analytical procedures and methods validation. Endereço:

<http://www.fda.gov/cber/gdlns/methval.pdf> .

[5] FDA (Food and Drug Administration), 1987. Guideline on general principles of process validation. Endereço: <http://www.fda.gov/cder/guidance/pv.htm>.

[6] FUKUMOTO, C.J. ; OLIVEIRA, C.A.F. Determinação de mercúrio em pescado comercializado no município de São Paulo, SP-Brasil. Higiene Alimentar. v. 9, n. 40, p. 27-30, 1985.

[7] JOHN, M., 2000. Inductively Coupled Plasma Optical Emission Spectrometry \& Flame Emission Spectroscopy. New York. Endereço:

$<$ http://cs.alfred.edu/ maurojc/papers/pdf/icpfes.pdf $>$.

[8] GINE, M.F. Espectrometria de Emissão Atômica com Plasma Acoplado Indutivamente (ICP-OES). Piracicaba: CPG/CENA. 1998, 143p.

[9] KITAHARA, S.E.; OKADA, I.A.; SAKUMA, A.M.; ZENEBON, O.; JESUS, R.S.; TENUTA-FILHO, A. Mercúrio total em pescado de água doce. Ciênc. Tecnol. Aliment., v.20, n. 2, p. 267-273, 2000.

[10] PEREIRA, O.M.; HENRIQUES, M.B.; ZENEBON, O.; SAKUMA, A.; KIRA, C.S. Determinação dos teores de Hg, $\mathrm{Pb}, \mathrm{Cd}, \mathrm{Cu}$ e $\mathrm{Zn}$ em moluscos (Crassostrea brasiliana, Perna perna e Mystella falcata). Revista do Instituto Adolfo Lutz. v. 61, n. 1, p. 19-25, 2002.

[11] PETERSON, C.L.; KLAWE, W..L.; SHARP, G.D. Mercury in tunas: a review. Fishery Bulletin, v. 71, n. 3, p. 603613, 1973.

[12] PREGNOlatTo, W.; GARRIDO, N.S.; TOlEDO, M. Pesquisa e determinação de mercúrio em peixes de água doce e salgada. Revista do Instituto Adolfo Lutz. v. 34, n. 1, p. 95-100, 1974.

[13] SILVA, A. M. Toxicologia do mercúrio no meio ambiente. São Paulo: Cered. 1951, 26p.

[14] WHO (World Health Organization). Environmental health criteria for methyl mercury. Geneve, International Programme on Chemical Safety. 1990, 144p.

[15] YAllOUZ, A.; CAMPOS, R.C. Niveis de mercúrio em atum sólido enlatado comercializado na cidade do Rio de Janeiro. Ciênc. Tecnol. Aliment. v. 21, n. 1, p. 1-4, 2001. 